\title{
What You See Is What You (Can) Get? Designing for Process Transparency in Financial Advisory Encounters
}

\author{
Philipp Nussbaumer and Inu Matter \\ University of Zurich, Department of Informatics, Binzmuehlestrasse 14, \\ 8050 Zurich, Switzerland \\ \{nussbaumer, matter\} @ifi.uzh.ch
}

\begin{abstract}
In this paper, we report on a study to establish process transparency in service encounters of financial advisors and their clients. To support their interaction, we implemented a cooperative software system for tabletops, building on transparency patterns suggested by the literature. In evaluations, however, we found that our design did not improve the perceived transparency and comprehensibility. Introducing the IT artifact into advisory failed to enhance the client's overall experience and even seemed to negatively influence the client's perception of the advisory process. Using the representational guidance of depicting the process and its activities as a navigable, interactive map made clients believe that interactions with their advisor were restricted to the system's functionality, thus expecting that what they see is all they can get.
\end{abstract}

Keywords: process transparency, collaboration, advisory, tabletops.

\section{Introduction}

In the changing market environments of the last several years, an increasing number of financial service providers (FSPs) has turned to individualized financial advisory services as a competitive differentiator. For Swiss banks, however, [1] found that implementation and application of such services are still in their infancy and that customers are inherently dissatisfied with their FSP's service provision. A main point of criticism thereby is related to a lack of transparency and comprehensibility of advisory services, i.e., the activities performed therein, their interrelations as well as their results. In service encounters, the client often perceives her advisor as a "black box", collecting client information as an input and returning investment recommendations as an output, usually without revealing a consistent line of reasoning. Thus, it is difficult for clients to relate their voiced needs (i.e., their problem space) to the advisor's recommended strategy and products (i.e., the solution space) [2]. Additionally, the information asymmetry between the actors (with the advisor typically being more knowledgeable) and the potential interest asymmetry (advisors exploiting information asymmetry to take advantage on their clients) lead to further distrust and thereby impact client satisfaction.

In this paper, we argue that establishing process transparency in service encounters may alleviate these issues, i.e., allow for client understanding and comprehensibility 
while decreasing information and interest asymmetries. The notion of process transparency has been discussed in different research fields with various meanings and levels of concreteness. A suitable conceptualization can be found in organizational literature, where process transparency is often related to internal and external process communication. As such, transparency has been argued of being related to customer satisfaction, as the transparent communication of processes enables clients to appreciate the company's activities and efforts as well as to identify their role in service provision. On a more specific level, in CSCW research, process transparency corresponds to communication processes in work groups and has been shown to influence the organization of communication and cooperation processes (e.g., [11], [12], [13]).

To support process transparency in service encounters based on these notions, we designed a collaborative tabletop artifact providing visualization and simulation functionality for the main activities of investment advisory, prominently featuring a navigable depiction of the process and its activities (e.g., needs elicitation, risk analysis, definition of investment strategy). The design's rationale was to present and allow control of the organization's advisory process and its activities, thereby mediating advisor-client communication and furthering the client's understanding.

To measure the effects of increased process transparency on the interaction of client and advisor as well as the client's understanding and satisfaction, we compared the traditional setting (advisor using pen \& paper) with the IT-supported setting (advisor and client cooperatively using the tabletop artifact) in controlled within-subject evaluations. Surprisingly, perceived transparency and comprehensibility as well as satisfaction were rated the same or even lower for the IT-supported setting compared to the traditional setting.

We found several explanations for the design failing to improve the client's overall experience. Most prominently, we found that clients felt rather restricted by the system, taking the depiction of the process and its activities as the boundaries of advisory along the lines of "if you cannot see it, you cannot get it". Despite of having based our design considerations on comprehensive explorative research and having evaluated the resulting designs with domain experts, we underestimated the side effects of process transparency on advisor-client interaction.

\section{Transparency Issues in Financial Advisory}

Today, the most promising strategy of financial service providers to differentiate against competitors is to offer highly personalized services. These cannot easily be compared or imitated due to their dynamics and complexity [3]. However, since the fundamentals of such services have not yet been established, FSPs have been counteracting cost pressure by optimizing their advisory services towards efficient and effective product sale rather than individualized advisory. As a consequence, the quality of advisory services has been perceived as rather dissatisfying or even inappropriate for customers (e.g., [4], [1], [5], [6]).

In an exemplary investment advisory consultation of a Swiss bank, the client and the advisor meet in a designated consultation room. In the case of prospect clients, they meet for the first time, so the advisor has minimal information about the specific needs of his vis-à-vis. Thus, for the first few minutes he will engage in small talk to 
gather basic information about the client (financial situation, needs and wishes), taking notes on his notepad. The advisor then typically presents the bank's generic advisory process, which he will use to optimize the client's financial situation and to help her in achieving her goals. Throughout the remainder of the encounter, the advisor tries to gather as much information about the client's financial situation, her risk preferences, investment experiences as well as her interests in particular asset classes. Building upon this information, he will then suggest an investment strategy that proportionally attributes the client's investment to different asset classes (e.g., shares, bonds, money market). After some iterations of adapting this strategy to the client's preferences (e.g., increasing the amount of bonds and decreasing the amount of shares), the first encounter is finished (typically after up to 90 minutes). The advisor will propose to prepare a product portfolio for the agreed strategy, which will be either sent to the client (including material for establishing the contract) or discussed in a subsequent encounter.

Regarding such an advisor-client interaction, several characteristics can be found that are detrimental to perceived advisory quality. Most prominently, such encounters are inherently impacted by information asymmetry and interest asymmetry, problems that are well established in scientific literature in context of the 'principal-agent problem' [7, 8]. Information asymmetry results from the customer being generally less knowledgeable than the advisor - thus, she cannot be sure whether the advisor actually gathers and provides all relevant information and recommends appropriate solutions for her financial needs. The relation between customer and advisor can be additionally strained by conflicts of interests. Advisors might exploit information asymmetry by, e.g., superficial information gathering and provision or, even worse, recommending products that are unsuitable for the specific customer's needs but profitable in terms of fees.

In a comprehensive study of advisory practice in Swiss banks, [1] found that clients are quite aware of these problems. As a result, they do not consider financial advisors as being very trustworthy - also, they perceive the advisor's knowledge about market trends or even the bank's products to be rather limited. All in all, clients are not very confident that financial advisors present adequate solutions to their needs. Many of the problems found in advisory encounters relate to a perceived lack of transparency, i.e., the absence of comprehensible and coherent advisory schemes that allow verification of the progression and results. Not recognizing the underlying rationales of advisory and its activities, clients also perceive advisory encounters as lacking personalization [1].

\section{Design of the Prototype System}

Analyzing the status quo of financial advisory from interviews and discussions with advisors and clients (including interviews of 21 advisors from 19 FSP, client focus groups totaling 28 participants as well as a client survey with 136 participants; see [1]), we designed problem scenarios of typical financial advisory encounters to derive generic design considerations for comprehensible and interactive service encounters between advisor and client [9, 10], including the notions of cooperation, process transparency, information transparency as well as cost transparency. 
In the following, we will focus on the concept of process transparency. Building on the issues identified in our field work, we define process transparency as the degree of the client being able to follow and comprehend the performed activities (what constitutes an activity and why is it performed) and their succession in advisory. We will discuss the according requirements, the designs we chose to satisfy them as well as their implementation for a multi-touch tabletop device.

\subsection{Design Requirements}

From discussions with advisors and clients and observations of work practice, we derived the following basic requirements to enhance transparency of advisory encounters:

DR.1 Process awareness: Clients commented about the advisor being a "black box", i.e., interaction between advisor and client being somewhat unpredictable regarding the information gathered by the advisor and how they influence the output of advisory. We therefore suggest making the process of advisory visible, i.e., increasing the client's understanding by transparently presenting the performed activities and associated actions. This also involves structuring the succession of activities and specifying defaults for their execution.

DR.2 Process adaptability: In discussions with advisors and managers, we found that advisors do not favor the rather rigid process guidelines, which organizations try to establish in their efforts to standardize advisory activities. Processes and activities visibly mediating advisor-client interaction therefore have to be adaptable, i.e., they must feature multiple starting points and offer the possibility to change their order; thereby, both the advisor and the client should be enabled to keep track of the progress of the advisory process.

DR.3 Shared information space: Addressing the information and interest asymmetry between advisor and client requires the provision of transparent information access for both parties. This shared information space should support communication and interaction of advisor and client while also allowing for process awareness and adaptability as discussed above.

\subsection{Design Rationales}

Research on Computer Supported Cooperative Work (CSCW) and Computer Supported Collaborative Learning (CSCL) has been engaged in applying mechanisms of process transparency to support and enhance organization of communication processes. Almost twenty years ago, [11] already stated that "computer support of cooperative work should aim at supporting self-organization of cooperative ensembles as opposed to disrupting cooperative work by computerizing formal procedures" (p. 17). They acknowledged, however, that the organizational models should be made accessible to the users by the system, supporting the user in interpreting procedures and evaluating their rationales and implications.

For group work, [12] argues that the underlying communication and cooperation processes are not always clearly defined and comprehensible and thereby lack transparency regarding the status of the group work relative to the overall process; in such 
cases, failing to establish process transparency may have undesirable outcomes on communication [13]. For similar reasons, research also suggests that members of computer-supported work groups have to explicitly agree on communication and cooperation processes [14].

External representations have been studied in the context of learning and problem solving tasks, showing that the nature of representation may influence the conception of the problem and hence ease the finding of an appropriate solution (e.g., [15], [16]). For collaborative processes in learning, [17] investigated the influence of representational guidance by comparing different tools for constructing representations of evidential models on collaborative learning processes and outcomes. They argue that external representations play at least three roles in situations of groups using shared representations in constructive activities (p. 473):

(1) Initiating negotiations of meaning: when constructing or manipulating shared representations and trying to obtain agreement, members of a group have to explicate and negotiate the representations' meanings and their shared beliefs.

(2) Being a representational proxy for deixis: collaboratively constructed representations may serve as an easy way for participants to refer to previous ideas and facilitate subsequent negotiations, thereby increasing the conceptual complexity that may be handled in a group's interaction.

(3) Enabling implicitly shared awareness: shared representations may also serve as an external memory of the collaborating group, reminding the group members of previous ideas and comments.

Testing different representations with pairs of participants, [17] found that representations have impacts on learners' interactions and may differ in their influence on subsequent collaborative use of the knowledge being manipulated; thereby, visually structured representations can provide guidance for collaborative learning that is not afforded by plain text. They also speculate that graph representations will be most useful for gathering and relating information.

In [18] two approaches of establishing representational guidance are discussed, varying in their degree of process structure. Maps strive to only provide a basic means of orientation while not constricting the actual enactment of a process. In [19] such an approach was used in the context of process knowledge learning; they provided their students with a graphical hypermedia-based process representation to support cooperative process enactment; the representation contained associated materials with which the users could interact while systematically carrying out the process they were learning. In [20], Carrell et al. found that the usage of graphical process models during the preparation of collaboration leads to more knowledge exchange and integration and stronger individual and collaborative use of the software platform.

Kienle [18] uses diagrammatic representations for a software system supporting collaborative learning processes. She thereby suggests that navigable models are apt to give orientation and structure in the sense of representational guidance; as the model is always present for the user, it also should be internalized more easily.

In contrast to maps, which have the goal to give only basic orientation of communication and cooperation processes, (cooperation) scripts consist of detailed instruction sets of how a group should interact and collaborate to solve a given task. Being highly structured, such processes (and their representation in software systems) are 
rather inflexible and run the risk of not fitting the processes already established in groups, which in turn might lead to non-acceptance [21].

Similar to [18], in our design we follow the map concept and address process awareness (DR.1) by depicting the advisory process as a fixed-positioned, navigable diagram, which is always present and can be controlled by both the advisor and the client (see Figure 1). As such, we also built our design on the different roles of external representations discussed by [17], enabling the advisor and client to discuss and reflect upon the process, allowing them to select and revisit activities at any time and track progress (DR.2), as well as helping to establish a shared information and activity space (DR.3) that may serve as an external memory. To enhance the system's affordance for the users, we based the information design (e.g., pie charts, risk-returngraph; see Figure 1) on actual information material used for investment advisory in Swiss banks.

In contrast to the representations discussed by [12, 18] and [17], however, the design does not allow manipulating or changing the representation of the process itself. This is due to mainly two reasons: advisory processes typically consist of three


Fig. 1. Basic design of the system's front end 
generic process steps, i.e., (1) capturing and discussing the current situation, (2) finding and configuring optimizations, (3) implementing the optimizations; these steps are related to specific activities, which are generally accomplished in a specific order (e.g., for the optimization phase: discussing the client's risk profile and constructing a suitable strategy). Also, most of the activities found in financial advisory are interdependent (e.g., the strategy being a function of the client's risk profile and her preferences, which are discussed in the first and second process step). Thus, configuring these specific process steps and activities regarding their order might not be meaningful in most cases.

Secondly, the advisory processes are subject to compliance (standardization) as well as legal regulations (due diligence), constraining an advisor's freedom to "skip" activities. From the client's point of view, we also wanted to depict the "standard" process and its associated activities, as to give her an overview of the different advisory steps and associated activities. We did, however, consider it to be important that the advisor and client could themselves decide on the order of activities, allowing them to use the system's functionalities as needed. Therefore, the design allows ignoring interdependencies of activities, e.g., permitting to create an investment strategy without having configured the client's risk profile.

(1) Navigable process map, indicating the current process step and activity as well as the overall progress: the highlighting indicates that the current activity is associated to finding an appropriate investment strategy (asset allocation) based on the information gathered in the previous steps.

(2) Shared information/activity space: in the current activity (definition of investment strategy), the shared information space allows the advisor and client to collaboratively specify and adapt the client's asset allocation (interactive pie chart on the bottom right) while simultaneously illustrating the impacts on risk and return (graph on the bottom left).

(3) Advisor "Cockpit": shortcuts to navigate the process and its activities.

(4) Client "Cockpit": allows the client to display additional information for the specific activity as well as to access the projected asset growth based on the entered information (as depicted in Figure 2).

\subsection{The SurFinance Prototype}

To implement our prototype, we decided to use a multi-touch tabletop device (Microsoft Surface), so the advisor and client could simultaneously interact with the shared information and activity space without explicit handovers. Also, equipping a situation previously being equipped with pen $\&$ paper only, we assumed that a tabletop would be perceived as less intrusive and less disruptive for social interactions. In the newly designed encounter, the actors seat themselves at the tabletop device (see Figure 2 for an overview), which supports them in accomplishing the most important and complex activities (needs elicitation, risk profiling, strategy development, product selection).

While engaging in initial small talk, the advisor is enabled to transparently add the client's needs into an area at the center of the screen, assuring the client that her wishes and needs are taken seriously. To stimulate the client in thinking of additional needs and wishes, pictograms of basic categories (planned purchases, education, and housing) are readily available. Wishes and needs may be detailed with costs and 
contextualized with a timeline to express the desired period of goal fulfillment. Using the client's financial information, a projection of the potential growth of wealth is added to the timeline, allowing for an assessment whether the client's goals may be accomplished. In such discussions, the advisor acts as a coach, who strives to enable the fulfillment of the client's needs by mapping them to appropriate financial strategies and products.
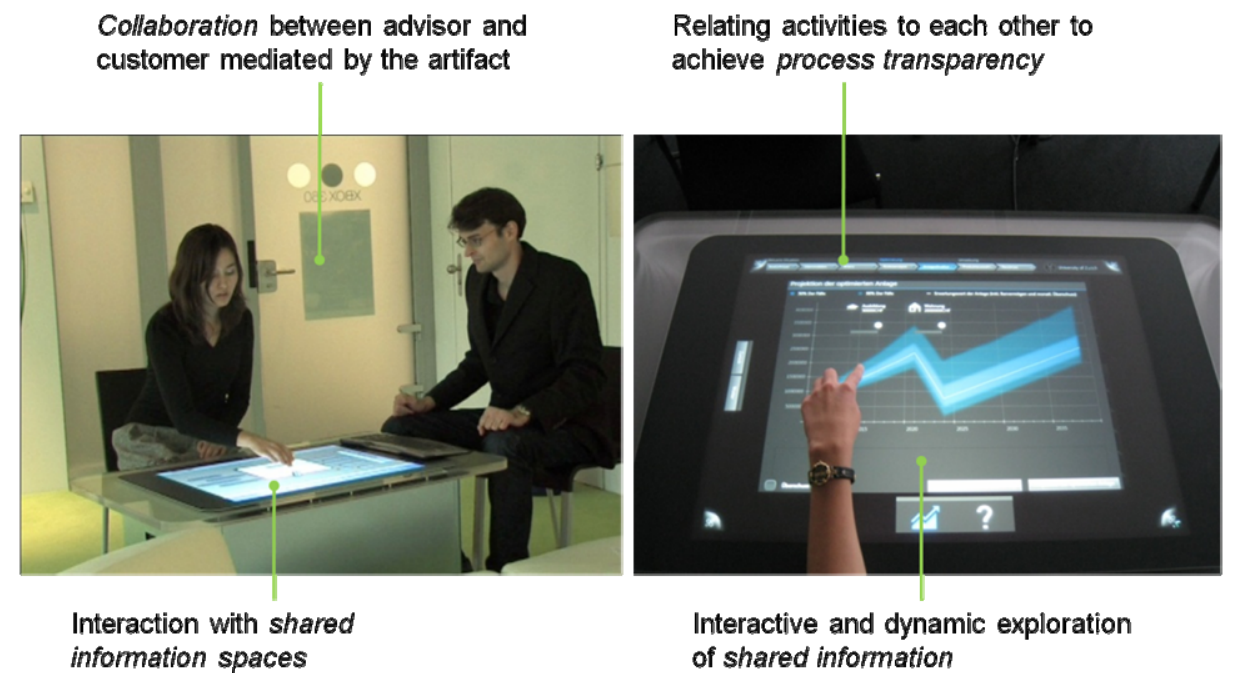

Fig. 2. SurFinance prototype

Collaboratively using the artifact, client and advisor are enabled to jointly define investment strategies that transparently include the defined needs and goals. The dynamic visualization enables the advisor to comprehensibly argue for or against specific strategies, while the client can immediately track the impacts on her financial situation. As an overview of all performed activities is provided at any given time, the client may also refine and revise her data by directly navigating to the specific activity. Having agreed on a strategy, the client and advisor may directly implement it by selecting appropriate products, or the advisor - similar to the traditional setting - may prepare an appropriate portfolio for a follow-up encounter.

\subsection{Hypotheses}

Based on the previous discussion and our experience from exploratory research [1], we state the following hypotheses.

One of the main design goals of our prototype is to depict the advisory process and visualize its activities, allowing the clients to actively interact. We hypothesize that these novel possibilities increase the client's comprehensibility and understanding of advisory's contents and activities. We thereby implicitly assume that in traditional advisory situations - having to rely on the advisor's explanations and drawings - it is 
difficult for clients to establish a detailed understanding. The according hypothesis reads as follows:

\section{H1.1: Using an IT artifact enabling process transparency, the comprehensibility of the order of activities as perceived by the clients is higher than that of the tra- ditional financial advisory encounter.}

With our prototype, we aim at the client becoming an integrative co-producer of the advisory result by contributing to and interacting in activities, while immediately being able to assess their intermediate results. We therefore hypothesize that using the artifact increases the client's understanding of how and why the results came about:

H1.2: Using an IT artifact enabling process transparency, the comprehensibility of the results as perceived by the clients is higher than that of the traditional financial advisory encounter.

In focus groups and interviews, clients often argued that advisors tended to recommend their "standard" products without the clients being able to influence their decision making. We therefore hypothesize that the presence of an interactive, shared artifact should increase the perceived degree of being able to influence the process and its results compared to the traditional situation, which in turn should be perceived as more restrictive:

H2.1: Using a shared IT artifact enabling process transparency, the degree of being able to influence the solution finding process as perceived by clients is higher than that of the traditional financial advisory encounter.

In addition to a higher degree of influencing the process, we hypothesize that in the IT-supported setting the client is better enabled to actively participate in activities:

H2.2: Using a shared IT artifact enabling process transparency, the client perceives a higher possibility to participate in activities than that of the traditional financial advisory encounter.

As discussed above, surveys show clients being inherently dissatisfied with advisory services of their banks. Having identified transparency issues as a possible cause, we suggest that introducing IT-enabled process transparency should also positively affect the clients' satisfaction with the advisory encounter.

H3: Using a shared IT artifact enabling process transparency, the client's overall satisfaction is higher than that of the traditional financial advisory encounter.

\section{Experimental Evaluation}

We built our SurFinance prototype in two iterations. The first prototype was implemented by a group of four students as part of a Master's project, and already featured the basic transparency design reported in Section 3. To evaluate our design rationales, we discussed the prototype and its underlying concepts with representatives of four major Swiss banks. Their unanimously positive feedback encouraged us to enhance and functionally extend our prototype in order to evaluate it with real users. To get directions in revising the prototype and finding additional functional requirements, we conducted three focus groups of 15 domain experts in total (one focus group for 
financial advisory experts, financial software developers and design experts each); based on their input, we built the second iteration of the prototype. In the following, we will report on the experimental evaluation of this prototype (as described in Section 3.3).

\subsection{Experimental Design}

The evaluation involved 12 clients, each of them performing an investment planning task in two different settings in a within-subject design. The participants were recruited by convenience sampling through postings on a university forum (each received 40 $\mathrm{CHF}$ ), eight of them being university students of various study programs. The participants were between 21 and 50 years of age, with high proficiency in computer use (6 participants categorized themselves as being professional users, 5 as advanced users and only 1 participant reported to use IT only occasionally). Half of the participants were female, and five participants already were experienced with advisory.

Clients received a short introduction before the test sessions, including explanations about their time table, instructions about their task and financial profile. The planning task involved the investment of a specific amount of money from 250'000 to 480'000 CHF, while considering specific wishes and goals, e.g., purchasing an apartment. The clients were allowed to keep back their information until the advisor explicitly asked for it. To preserve the participants' privacy, they were provided with a profile that included key figures of their assumed financial situation. Each client participated in two test settings. One setting corresponded to the traditional (pen \& paper) advisory that is typically provided in Swiss banks, the other setting involved the use of the SurFinance prototype. The participants were randomly assigned to either start with the traditional or the IT-supported test setting (50\% of participants each). Test sessions of each setting were limited to 30mins.

The sessions were conducted by four financial advisors (three being male, one being female) of a Swiss bank. Their age was between 31 and 40 years. They had been practicing their job as financial advisors for four up to seven years. All of them classified themselves as being advanced IT users. Each of them received a 30mins handson training with the prototype system. In their briefing, the advisors were given two main instructions:

(1) In the traditional test session (without IT), they were asked to perform advisory along their actual practice. Advisors were allowed to use all material they needed to advise a prospect client asking for support in investment planning. Prior to the evaluation, we therefore asked them to bring the according material with them.

(2) In the test session featuring the SurFinance prototype, the advisors were required to use the artifact at least once in their session (providing interaction possibility for the client) but were free to decide at which point they would introduce the tool and in what order they would perform specific activities (need elicitation, risk profiling, etc.).

Each advisor performed three traditional advisory sessions (without IT) as well as three IT-supported sessions using the SurFinance prototype. 


\subsection{Data Collection}

The tests were conducted on two days at the end of July and beginning of August 2010. After their trials, clients received a quantitative questionnaire and were debriefed in semi-structured interviews (having an average duration of 30mins); advisors were asked to provide qualitative feedback in semi-structured interviews (average duration of 60mins). The semi-structured interviews covered the following main aspects: disturbing moments, comprehensibility of the advisory sessions, collaborative activities and interactive moments, advantages and disadvantages, preferences regarding the advisory setting (traditional vs. IT-supported) and additional comments.

Our quantitative questionnaire included items to test our hypotheses as well as demographic items (age, gender, education, advisory experience, IT skills). As we are not aware of any standardized items for comprehensibility (H1.1 and H1.2) of (advisory) processes and the succession of activities, we measured comprehensibility with the following two items:

- "I could understand at any time why the activities of the advisory session were following a specific order." (Comp1)

- "I do understand how the results of the advisory session have been achieved." (Comp2)

To investigate the client's perceived influence on the advisory process $(\mathrm{H} 2.1)$ and on her ability to perform actions (H2.2), we used the following two items:

- "Overall, I was able to influence the solution finding process of the advisory session." (IoAP)

- "Overall, the advisory situation enabled me to participate in activities." (PA)

For hypothesis H3 we used items of the Yield Shift Theory of satisfaction (Briggs et al. 2008).

Each item was measured once for each advisory session (traditional and IT-supported advisory) with a seven-point Likert-scale (from $1=$ "I strongly disagree" to $7=$ "I strongly agree").

\subsection{Results}

The data from our evaluation were tested with two-sided t-test for paired samples with differing variances.

The perceived comprehensibility of the order of activities (Figure 3a; avg. traditional setting $(\mathrm{TS})=5,08$; avg. IT-supported setting $(\mathrm{ITSS})=5,00$ ) as well as the comprehensibility of the results (Figure $3 b$; avg. TS $=5,42$; avg. ITSS $=4,58$ ) were rated lower for the IT-supported setting than for the traditional setting. The t-test, however, did not show any significant difference between the two settings. We can therefore neither support nor falsify hypotheses H1.1 and H1.2.

At the debriefing interviews, clients brought forward several reasons for their ratings on comprehensibility. Two participants found that the advisor's explanations regarding the process and its activities were better in the traditional setting than those received in the IT-supported setting. They reported that it was unclear to them how the charts and results of the IT artifact came about. Advisors also reported to having 
had difficulties in explaining visualized information, especially when charts contained multiple information dimensions.

One client and one advisor perceived the conversation's pace and progress of the IT-supported setting as too fast. Another three clients experienced "information overflow"; while one was overwhelmed by the amount of information channels (ITartifact and advisor), another found that the IT artifact required too much knowledge. Advisors pointed out that this resulted in clients asking more specific questions, which were difficult to answer because of the clients' lack of knowledge.

In the traditional setting, two clients reported to be overwhelmed by information (e.g., investment possibilities) provided by the advisor.

Five clients argued that in the traditional setting the conversation with their advisors was more consistent and "smooth" as opposed to the IT-supported setting, where conversation was "interrupted" by the use of the IT artifact.

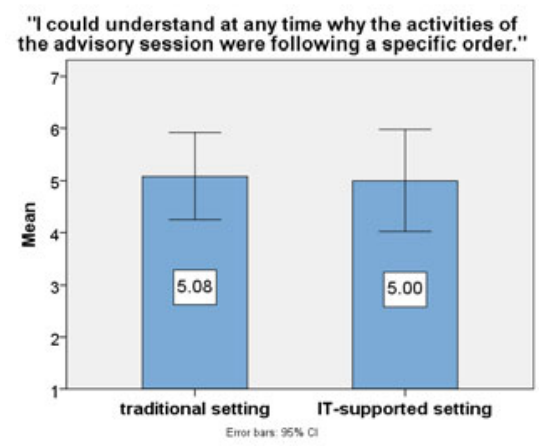

Fig. 3a. Mean Comp1 (error bars: 95\% CI)

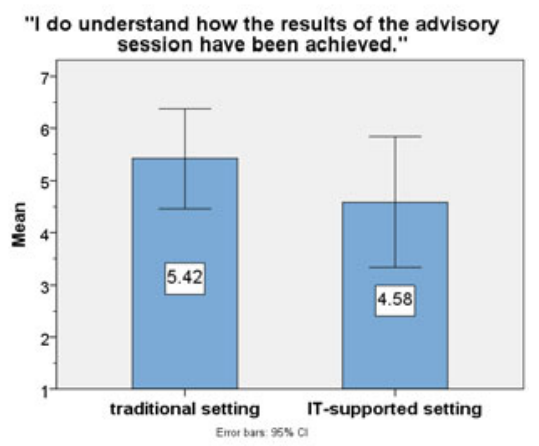

Fig. 3b. Mean Comp2 (error bars: 95\% CI)

Overall, seven clients perceived the comprehensibility of the different settings as being equal, while one preferred the traditional setting and another one preferred the IT-supported setting. Five clients and three advisors, however, explicitly stated that the artifact's visualizations were greatly supporting comprehensibility and transparency. Additionally, one advisor appreciated the navigable process map as an external memory.

Figure $4 \mathrm{a}$ and Figure $4 \mathrm{~b}$ show the means of the clients' perceived degree of being able to influence the solution finding process (IoAP; avg. TS =5,50 and avg. ITSS = 4,00 ) and the clients' perceived possibility to participate in activities (AP; avg. TS = 5,17 and avg. ITSS =4,50). Again, for both items the average agreement is lower for the IT-supported setting. The results of the two-sided t-test indicate a significant difference between the two means (TS and ITSS) of IoAP $(\mathrm{p}<0.05$, $\mathrm{df}=11, \mathrm{t}=2,691)$ but no significant results for AP. Hence, our data do not support hypotheses H2.1 and H2.2.

For these results, we obtained the following explanations from the participants. Three clients believed that they could better influence the result of the advisory process in the traditional setting, while one was stating the opposite. Seven participants found that the traditional advisory was more personal than the IT-supported setting, some of them stating their impression that the advisor was focusing too much on the 
system. This argument was also raised by advisors, which found it difficult to maintain the conversation with the client while interacting with the artifact.

One client perceived the process in the traditional setting as being more flexible than in the IT-supported setting. Even though advisors were not obliged to overly use the system in the IT-supported setting (or use its functionalities in a specific order), they also found the system to restrict their performance.

Four clients perceived a higher possibility to influence and shape the activities in the traditional setting, three in the IT-supported setting and three claimed to have had the same possibilities in both settings. Only one client seemed to have realized that the order of activities could be changed by skipping activities or selecting previous process steps.

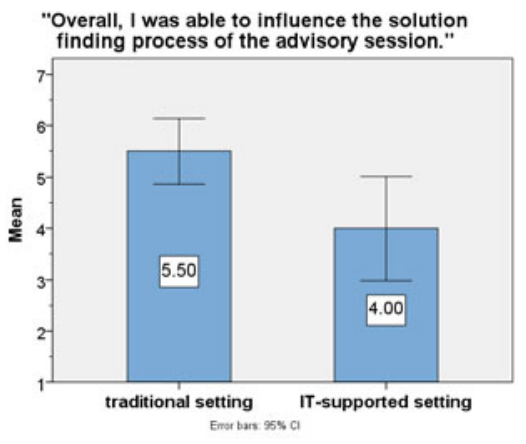

Fig. 4a. Mean IoAP (error bars: 95\% CI)

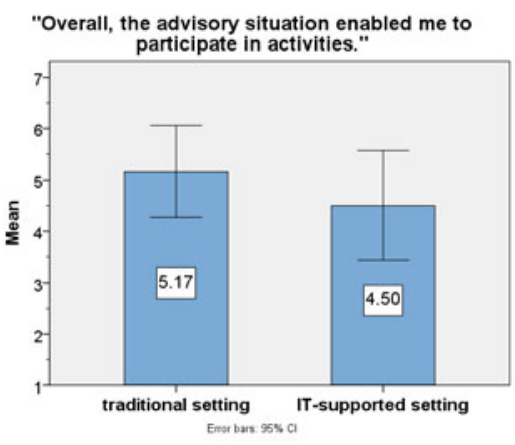

Fig. 4b. Mean AP (error bars: 95\% CI)

While five clients would have liked to interact with the system more often, five clients found their interaction with the system to be sufficient. Only one client, however, thought that he would disturb the advisor by interacting with the system. Interestingly, advisors had no reservations in letting the client interact with "their" system, perceiving the clients' activation as a benefit.

On average, satisfaction with the advisory situation (Figure 5) again was rated higher for the traditional setting than for the IT-supported setting (avg. TS $=5,37$; avg. ITSS $=4,90$ ), but the t-test reveals no significant difference. Thus, our data do not support $\mathrm{H} 3$.

In the interviews, six clients stated that they would prefer an IT-supported advisory in their next advisory session. Five participants preferred to have their next advisory encounter in a traditional session. Two clients and one advisor proposed to combine the strengths of both sessions (starting the advisory session with traditional face-toface conversation and consulting the IT artifact only later), highlighting the importance to maintain the advisor-client conversation.

Overall, eight clients would have recommended the IT-supported advisory to others, while five would have recommended the traditional advisory. Three of four advisors enjoyed using the IT artifact and were looking forward to have such tools at their disposal. The remaining advisor, however, felt insecure about using IT with his clients. 


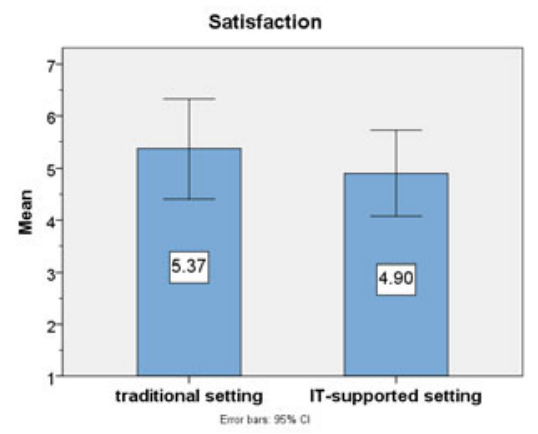

Fig. 5. Mean satisfaction (error bars: $95 \% \mathrm{CI}$ )

\section{Discussion}

Looking at the results, we can safely assert that our design for process transparency was not successful in improving the client's overall experience, with all investigated dimensions being rated lower for the new advisory setting. This, however, does not necessarily mean that the design per se was a failure. To the contrary, the clients' ratings of process transparency (comprehensibility of the activities and their results) were rather positive, though leaving room for improvement when being compared to the traditional setting. We suggest that the chosen design for process transparency might be ambiguous, thereby affecting and conflicting with other factors of advisory. For this conclusion we find various indications, which can be related to the system design as well as the introduction of technology into so far predominantly social situations.

Perceived authority and determinism: Though the implemented process structure was consistent with the traditional advisory's course of activities (to which advisors also complied in the traditional advisory scenario), both the advisors and clients found the process depiction to constrain their interaction. As such, the system was perceived to be authoritative and deterministic, imposing its process structure upon the users and restricting the user's control of the process. One client, for example, pointed out that the system could not provide a solution featuring a small amount of shares, whereas in the traditional setting the advisor just "took a note", thereby reassuring the client that he would bear that in mind. The process map - that we intended to provide basic orientation and structure - turned out to be conceived as a set of scripts, i.e., detailed instructions of how to interact and collaborate to solve the given tasks. Interestingly, when using the system, both the advisors and the clients seemed to feel obliged to stick to the process structure, scarcely changing the order of activities or omitting/revisiting them. Though this allowed the client to associate activities to clearly defined process steps and making their succession transparent, the overall interaction and control was unsatisfying.

Collaboration in advisory: Conceptualizing the interaction of advisor and client as collaborative work (in that they jointly solve the client's problem, being in need of 
each others' input to achieve their goals), we borrowed our mechanisms to establish process transparency from CSCW and CSCL research. We believe that in complex advisory situations like investment advisory, learning is an adamant cornerstone of a client's understanding and comprehensibility. In this context, however, the aspect of cooperation deserves some more attention. Research on CSCW and CSCL commonly assumes that work groups are pursuing a common and shared goal. In such work contexts, conflicts between participants are mostly related as how to achieve a goal, rather than related to what the goal is. In financial advisory encounters, nevertheless, the opposite might be true. The specific results of the service encounter - emerging from the information exchange between advisor and client - are difficult to anticipate before the actual consultation. Additionally, financial products are not only virtual but also credence goods, i.e., clients may not be able to demonstrate whether the success or failure of a purchased product is due to the counseling process, the advisor's (or bank's) efforts or is simply a product of chance. Thus, for the client the journey might actually be the reward - as the result may not easily (or objectively) evaluated, the process leading to a particular result will be closely scrutinized. As discussed above, clients being advised using the prototype system felt that it restricted their interaction with the advisor and concluded that what they saw was all they could get. The visualization of the process seemed to confine their problem space to the type of problems the system could tackle and, partly as a consequence, the advisor's solution space to a set of solutions for predefined problems.

Another influencing factor regarding cooperation can be found in the implicit changes of the participants' roles in the technologically supported setting. While the roles of advisor and client and their related (role) scripts [23] seemed to be clear in the traditional setting, the new, collaborative situation implicitly changed the existing role models. Using the cooperative artifact demanded stronger and more active inclusion of the client to accomplish the given activities. To the contrary, the advisors' role tended to change to "coach" the customer in finding the solution rather than providing it themselves.

Focus on the shared artifact: Contrary to the results of our survey, where clients voiced their distrust in banks and their advisors [1], the clients participating in our evaluation found the advisors to be likeable and empathic. The cooperative artifact with shared information spaces clearly shifted the users' attention from interpersonal communication to operating the system. While some clients did not like the idea of the advisor paying more attention to the system than to them, others appreciated the possibility to reflect on the visualizations and interrelations of the financial concepts without the advisor "constantly talking" to them.

In some activities, both advisor and client had problems in correctly interpreting the visualizations and expected interaction, leading to communication breakdowns. The advisor's misinterpretations may be attributed to the lack of training they received in using the system. For the client, however, not every breakdown was problematic - often, the visualization would raise further and more detailed questions. All in all, we speculate that the system's presence clearly interfered with the advisory situation, especially with the client-advisor interaction. 


\section{Lessons Learned}

Establishing process transparency in advisory encounters seems to be a double-edged sword - though possibly allowing for more comprehensibility and understanding of the process flow and its activities, disclosing the underlying mechanisms of advisory can negatively influence the clients' perception of controllability. In this paper, we introduced a design for process transparency that demonstrated such an effect. We argue, however, that the design's failure to consider such an influence provides several lessons to learn. In the following, we will discuss such lessons and show how we incorporated them into our next design iteration.

The depiction of the advisory process as a static, fixed-positioned map has proven to be a poor design choice. In contrast to cooperative work or learning efforts, which might profit from explicit representational guidance to negotiate and relate to a common course of action, in advisory encounters such process representations may lead to a perception of constrained control. In our follow-up design, we therefore abandoned the fixed-positioned process depiction in favor of an implicit illustration of activities and their interrelations. While the system still provides an overview of the process (regarding the basic phases of advisory without implying an order) that may be displayed if required, there are no fixed activities attached. The advisor may use all of the system's functionality in each process phase, whereby interrelations between the functionalities are visualized to support the client's comprehensibility and understanding. If advisor and client, for example, discuss an investment strategy using the system, only relevant information from other activities is displayed - thereby, the client learns about the impacts of activities and the decisions made therein, while the advisor is prevented from "skipping" relevant activities. Such a design of contextualized access to activities makes their relations transparent without forcing actors to use them.

For the users, visualizing the advisory process on the shared technological artifact seemed to imply that all activities have to be accomplished using the system. This clearly is not desirable, as specific activities such as discussing the client's personal needs and wishes or financial goals are better left to the face-to-face dialogue of the client and advisor. At some point of time, however, especially with increasing complexity, directing the dialogue from the inter-personal level to a more focused technology-mediated discussion might be helpful or even necessary (e.g., when contextualizing the client's financial goals with the projected performance of her investment portfolio). Such a situational use of the supporting artifact for specific activities requires smooth transitions from face-to-face conservation to focused interaction with the shared information space and vice versa. Such a requirement cannot be fulfilled by technology itself - therefore, we plan to accompany our follow-up design with practical recommendations for advisors of how to integrate the artifact into advisory while maintaining personal interaction and preventing too much focus on the artifact.

Finally, with all the functionality possibly being incorporated into a software artifact, process transparency design should also make clear that the artifact is not the advisor's replacement. The software system cannot (and, as discussed above, should not) account for all possible activities or special cases that might emerge in advisory encounters. Missing functionality or the system failing to meet a client's specific desires, however, must not lead to communication breakdowns. At this, we can learn from the advisors' present working practice - if the advisor is not prepared to 
immediately answer a question or fulfill specific demands, he will take a note and inform the client that he will take care of it later; the client is not expecting the advisor to be all-knowing - and she certainly should not expect it from the system.

\section{Conclusion}

In this paper we have discussed possible designs for process transparency in financial advisory encounters to increase the client's comprehensibility and understanding of the advisory process, its activities and its results. Drawing from CSCW and CSCL research, we used a fixed-positioned, navigable process map to support process orientation and highlight interrelations of activities. Though the comprehensibility and perceived transparency in using the prototype system are rated about as positive as for the traditional setting, our design failed to improve the client's overall experience. We argue that our design may indeed support transparency in advisory encounters, but may also have negative effects on other seemingly important dimensions, namely the perceived controllability and comprehension of the advisory process as well as the interpersonal relationship between advisor and client.

Acknowledgments. The research discussed in this paper is co-financed by the Swiss federal innovation promotion agency CTI.

\section{References}

1. Mogicato, R., Schwabe, G., Nussbaumer, P., Stehli, E., Eberhard, M.: Beratungsqualität in Banken. Solution Providers AG, Dübendorf (2009)

2. Novak, J.: Mine, yours...ours? Designing for Principal-Agent Collaboration in Interactive Value Creation. In: Proceedings of Wirtschaftsinformatik 2009, Wien (2009)

3. Buhl, H.U., Kaiser, M.: Herausforderungen und Gestaltungschancen aufgrund von MiFID und EU-Vermittlerrichtlinie. Zeitschrift für Bankrecht und Bankwirtschaft 20, 43-51 (2008)

4. Stiftung Warentest: Die Blamage geht weiter. Finanztest 8, 25-30 (2010)

5. Klöckner, B.W.: Beratertest. Peinliche Ergebnisse - große Chancen. Bankmagazin 56, 4243 (2007)

6. Evers, J., Krüger, U., Reifner, U.: Beratungsqualität in Finanzdienstleistungen. NomosVerl.-Ges., Baden-Baden (2000)

7. Golec, J.H.: Empirical Tests of a Principal-Agent Model of the Investor-Investment Advisor Relationship. The Journal of Financial and Quantitative Analysis 27, 81-95 (1992)

8. Eisenhardt, K.M.: Agency Theory: An Assessment and Review. The Academy of Management Review 14, 57-74 (1989)

9. Nussbaumer, P., Schwabe, G.: Gemeinsam statt einsam: Kooperative Bankberatung. Mensch \& Computer 2010, Duisburg (2010)

10. Schmidt-Rauch, S., Nussbaumer, P.: Putting Value Co-Creation into Practice: A Case for Advisory Support. Under Review (2010)

11. Schmidt, K., Bannon, L.: Taking CSCW seriously. In: Computer Supported Cooperative Work (CSCW), vol. 1, pp. 7-40 (1992) 
12. Kienle, A.: Integration of knowledge management and collaborative learning by technical supported communication processes. Education and Information Technologies 11, 161185 (2006)

13. Guzdial, M., Turns, J.: Effective Discussion through a Computer-Mediated Anchored Forum. The Journal of the Learning Sciences 9, 437-469 (2000)

14. Engeström, Y.: Activity theory and individual and social transformation. In: Perspectives on Activity Theory, pp. 19-38 (1999)

15. Larkin, J.H., Simon, H.A.: Why a Diagram is (Sometimes) Worth Ten Thousand Words. Cognitive Science 11, 65-100 (1987)

16. Zhang, J.: The nature of external representations in problem solving. Cognitive Science 21, 179-217 (1997)

17. Suthers, D.D., Hundhausen, C.D.: The effects of representation on students' elaborations in collaborative inquiry. In: Proceedings of the Conference on CSCL: Foundations for a CSCL Community, pp. 472-480 (2002)

18. Kienle, A.: Computerunterstützung für die Organisation menschlicher Kommunikationsprozesse. Habilitationsschrift, FernUniversität Hagen (2009)

19. Wang, W., Haake, J.M., Rubart, J., Tietze, D.A.: Hypermedia-based support for cooperative learning of process knowledge. Journal of Network and Computer Applications 23, 357-379 (2000)

20. Carell, A., Herrmann, T., Kienle, A., Menold, N.: Improving the coordination of collaborative learning with process models. In: Proceedings of the 2005 Conference on CSCL, pp. 18-27 (2005)

21. Dillenbourg, P.: Over-scripting CSCL: The risks of blending collaborative learning with instructional design. In: Kirschner, P. (ed.) Three worlds of CSCL. Can we support CSCL?, pp. 61-91. Open University of The Netherlands, Heerlen (2002)

22. Briggs, R., Reinig, B.A., De Vreede, G.: The Yield Shift Theory of Satisfaction and Its Application to the IS/IT Domain. Journal of the Association for Information Systems 9, 267-293 (2008)

23. Solomon, M.R., Surprenant, C., Czepiel, J.A., Gutman, E.G.: A Role Theory Perspective on Dyadic Interactions: The Service Encounter. The Journal of Marketing 49, 99-111 (1985) 